\title{
Comprehensive ablation study of near-IR femtosecond laser action on the titanium-based alloy Ti6AI4V: morphological effects and surface structures at low and high fluences
}

\author{
Dubravka Milovanović ${ }^{1, a}$ (D) Boris Rajčić $^{1}$, Sanja Petronić ${ }^{2}$, Aleksandra Radulović ${ }^{1}$, Bojan Radak ${ }^{1}$, \\ Biljana Gaković 3 , Marian Zamfirescu ${ }^{4}$, Catalina Albu ${ }^{4}$, and Jelena Savović ${ }^{3}$ \\ 1 Institute of General and Physical Chemistry, Belgrade, Serbia \\ 2 Department of Belgrade Polytechnic, The Academy of Applied Technical Studies Belgrade, Belgrade, Serbia \\ 3 VINCA Institute of Nuclear Sciences - National Institute of the Republic of Serbia, University of Belgrade, Belgrade, \\ Serbia \\ 4 National Institute for Laser Plasma and Radiation Physics - Bucharest, Magurele, Romania
}

Received 31 March 2021 / Accepted 9 November 2021 / Published online 5 January 2022

(C) The Author(s) 2021

\begin{abstract}
The surface of a titanium-based alloy Ti6Al4V was subjected to modifications by a near-IR femtosecond Ti:Sapphire laser, emitting at $775 \mathrm{~nm}$ pulses of $200 \mathrm{fs}$ duration, in single-pulse and multipulse regimes, with up to 400 accumulated pulses, and pulse energies ranging from 2.5 to $250 \mu \mathrm{J}$. The whole range of induced effects is presented, from gentle ablation and pattern occurrence to substantial crater formation. Very observable laser-induced parallel periodic surface structures are reported, appearing both within the damage spot area, with low fluences, and at the peripheries of the craters, with higher fluences - but also on crater walls, and inside the crater structures. Damage threshold fluences $\left(F_{\mathrm{th}}\right)$ and the incubation factor $(\zeta)$ were also determined.
\end{abstract}

\section{Introduction}

Due to their high specific strength, good hightemperature properties, good deformability and ductility, excellent corrosion resistance and great biocompatibility, titanium-based alloys are of great significance to biomedical applications, nuclear industry, aerospace and marine industry [1-8].

The surface of the material is significant in terms of the response of the environment to titanium and titanium-based materials. It usually has the form of a contaminated, oxidized surface layer, clearly not appropriate for various applications, requiring some surface modification. Also, titanium alloys exhibit low hardness and relatively low wear resistance which, again, commences on their surfaces [9]. Therefore, many surfacemodification methods have been used to improve the performance of these alloys.

Owing to the specific characteristics of laser light, the laser modification of metallic and alloy materials is highly competitive compared to other techniques [10] because of the non-contact, controlled and precise processing and modification of the surface [11-13]. The effects that occur during the laser action on the sur-

\footnotetext{
${ }^{a}$ e-mail: dubravka.milovanovic2007@gmail.com (corresponding author)
}

face of the material, among other things, depend on the duration of the laser pulse and the results of the action of short (ns and ps) and ultra-short $(<10$ ps to fs) pulses differ significantly. Usage of ultrashort pulses is characterized by a negligible contribution of thermal effects in the depth of the material, so they are most suitable for the formation of micro-/nano-size surface structures. Research of the surface modifications of the titanium and titanium-based materials by laser beams, especially with ultra-short pulses, is a field of research with significant impact on its application in nanotechnology and biomechanics.

Ti6Al4V alloy is one of the mostly used titanium alloys in biomedical applications due to its excellent combination of mechanical properties and chemical stability [14-17]. Therefore, it is of great research interest to devise a specific laser-induced modification of the alloy surface for specific applications [18-20]. The different laser fluence and number of accumulated pulses induce specific morphological changes on the Ti6Al4V alloy - from optical properties alteration, through laser-induced periodical and bump array structures, to deep craters. Laser-induced periodic surface structures (LIPSS, also called "ripples") are a laserinduced damage field phenomenon which can be generated on a various material-metals, semiconductors, dielectrics under different processing conditions, in a 
Table 1 Laser processing parameters

\begin{tabular}{ll}
\hline Pulse count & $1,2,5,10,50,100,200$ and 400 \\
\hline Pulse energy $(\mu \mathrm{J})$ & $2.5,5,10,25,100$ and 250 \\
Energy density $\left(\mathrm{J} \mathrm{cm}^{-2}\right)$ & $0.22,0.44,0.90,2.2,8.9,22.2$ \\
\hline
\end{tabular}

wide range of pulse durations (from continuous wave irradiation down to a few femtoseconds) [21,22]. One of the earliest investigations of LIPSS formation is reported by Birnbaum where he explains the formation by a diffraction effect and suggested that the surface relief is formed by material removal at the maxima of the electric field intensity. Emmony et al. as well as Keilmann and Bai proposed that LIPSS are originated from an interference of the incident radiation with polaritons of the surface bound to and propagating along the irradiated surface [23-27]. Periodic surface structures (PSSs), among others, are important in improving biocompatibility of titanium alloys, i.e., they increase the surface area, which in turn stimulates growth of osteoblasts [28]. This creates a continuous interest in the research that contributes to understanding how laser beam parameters affect the morphologically features that occur during the laser surface treatment.

The present paper represents a femtosecond laser modification study of the Ti6Al4V surface, the effects of a range of laser pulse energies of 200 fs laser pulses (single-pulse and multi-pulse) on the Ti6Al4V surface in air, in terms of surface structuring, ablation, ablation efficiency, threshold fluences and incubation factor. The main motivation was to obtain practical data in as wide a range as possible and under the most common conditions.

\section{Experimental}

A femtosecond Ti:Sapphire laser (Clark CPA-2101) was used for Ti6Al4V surface modifications. Laser processing was performed in air by a focused $(f=75 \mathrm{~mm})$ laser beam of $775 \mathrm{~nm}$ wavelength, repetition rate $2 \mathrm{KHz}$ and pulse duration of $200 \mathrm{fs}$. The samples were positioned in a horizontal $\mathrm{X}-\mathrm{Y}$ plane, with an accuracy of $1 \mu \mathrm{m}$. A CCD camera was used for visualization and focalization.

The pulses were accumulated from a single pulse to 400 pulses per surface spot. The pulse energies ranged from 2.5 to $250 \mu \mathrm{J}$, in increments as given in Table 1 . A series of dots were created by laser ablation on the sample surface by changing the number of pulses and laser energy. The pattern had a matrix arrangement, as shown in Fig. 1a. For statistics, each matrix was a group of $3 \times 3$ spots ( 9 spots) or $2 \times 2$ spots ( 4 spots) created with the same energy and number of pulses (Fig. 1b).

Characterization of the spots in terms of surface effects and changes in surface morphology was performed by scanning electron microscopy (SEM) (model
JOEL-JSM-6500F and model JOEL-JSM-6460LV). Surface parameters and topography analyses were done using a non-contact optical profilometry microscope (white light optical profiler Zygo NewView 7100).

\section{Results and discussion}

Laser-material interaction depends on the laser wavelength, pulse duration, pulse count and properties of the material. When an ultrashort laser pulse acts on a metallic target, many physical processes can be observed. Firstly, on a femtosecond timescale $(<100$ fs), laser radiation is absorbed by free electrons, and, as a result, strong non-equilibrium occurs between electron temperature and temperature of the lattice subsystem. Thermal melting, as a consequence of electronlattice temperature relaxation occurs within several to a hundred picoseconds, depending on the metal properties. Further on, the thermal stress induced by high temperature gradients can trigger plastic deformations, and they develop on a nanosecond timescale. Finally, cooling with resolidification of the metallic target takes place. Depending on the pulse energy, ablation and crater formation on the target may occur. All these processes are strongly correlated and can be described only jointly $[29,30]$. Material properties, laser wavelength, number of applied pusses, focus position, the target speed if it is movable, environment, etc., affect the appearance of the treated surface. The effects obtained presently were examined by optical microscopy, and particular details were then subjected to scanning electron microscopy and optical profilometry.

The amount of output energy per affected surface area, e.g., the laser fluence, $F$, was calculated using the equation $F_{\mathrm{p}}=\frac{2 \cdot E_{\mathrm{p}}}{\pi \cdot \omega_{0}^{2}}$ where $E_{p}$ denotes output energy of the laser pulse and $\omega_{0}$ represents the calculated beam radius at $1 / e^{2}$. The $\omega_{0}$ is usually calculated from the slope of the linear fit of the dependence $D^{2}=f\left(\ln E_{\mathrm{p}}\right)$, where $D$ denotes the spot diameter [30]. Since the intensity profile of the laser beam is Gaussian, but slightly elliptic, the irradiation parameters were calculated using the dependence $D_{1} \cdot D_{2}=f\left(\ln r m E_{\mathrm{p}}\right)$, where $D_{1}$ and $D_{2}$ are ellipsoid diameters. Calculated $\omega_{0}$ value for presented experimental conditions is $26.8 \mu \mathrm{m}$, and the subsequently calculated fluence values are presented in Table 1.

A selection of typical damage spots obtained presently is shown as SEM microphotographs in Figs. 2 and 3 It is obvious that the damage on the Ti6Al4V surface is more prominent with increasing pulse count and pulse energy.

The pulse counts of 1,2 and 5 , at pulse energies of 2.5 and $5 \mu \mathrm{J}$, and the corresponding fluences significantly below $1 \mathrm{~J} \mathrm{~cm}^{-2}$, can be arbitrarily considered "low fluence regime". Namely, we divided the results in two parts in accordance with term "gentle" ablation phase. Low fluence regime comprises irradiation with fluence above damage threshold that produces 


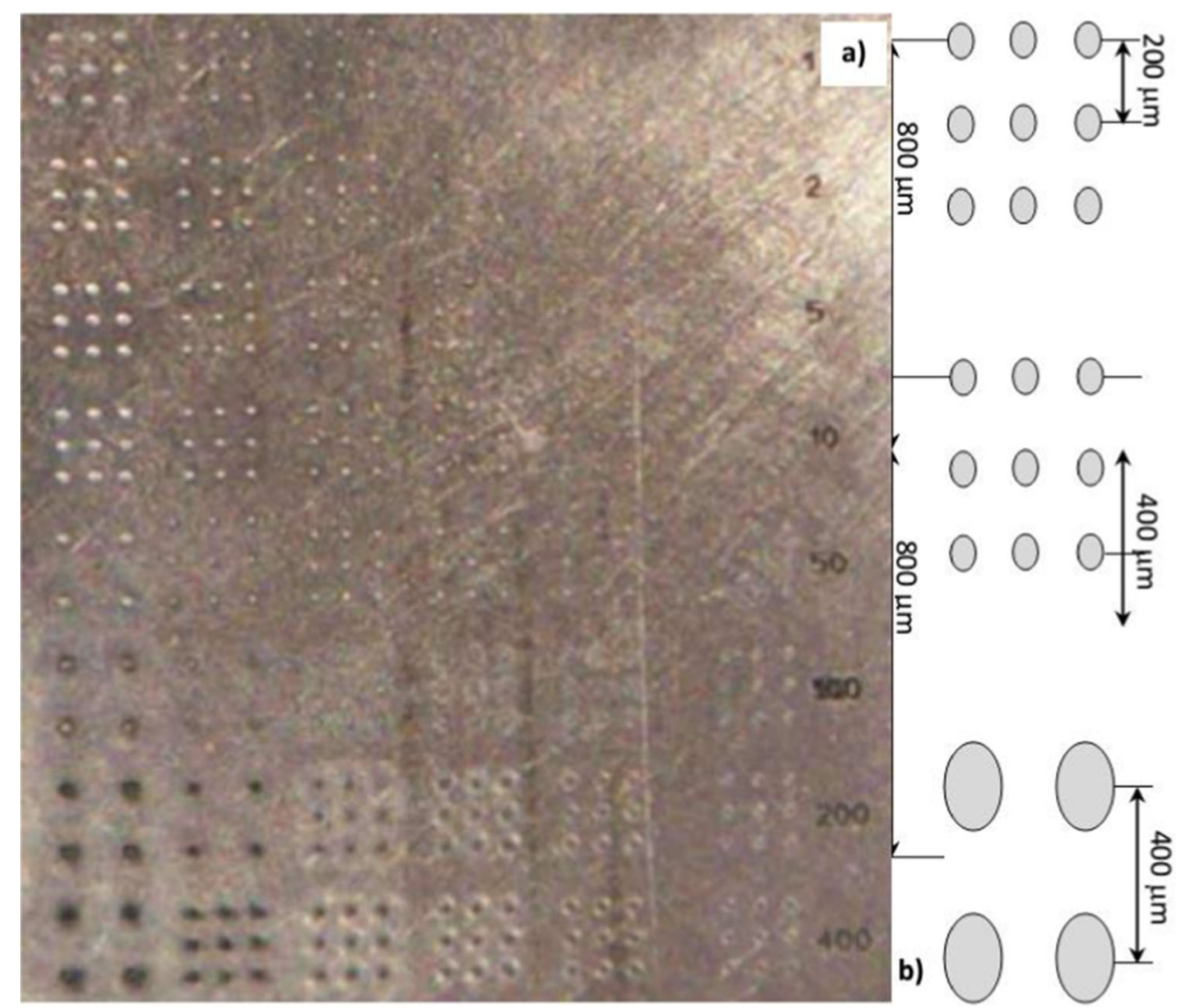

Fig. 1 a Ablated Ti6Al4V alloy surface, optical image, after 200 fs laser pulses at 775 nm wavelength, with increasing pulse energy and pulse count, as given in Table 1 , and $\mathbf{b}$ each spot in a matrix group of $3 \times 3$ spots $(9$ spots) or $2 \times 2$ spots (4 spots) created with the same energy and number of pulses

small morphologically changes and shallow craters, as observable in SEM micrographs [30]. In that regime, the laser/material interaction is characterized by uniformly distributed changes in topography, specifically (i) changes of surface roughness, without evident hydrodynamic effects (Fig. 3c1-c20, (ii) some form of selforganization and parallel periodic surface structures in the center of the damaged area (Fig. 3a2-b2) and (iii) laser-induced parallel periodic surface structures toward the periphery of the damaged area (Fig. 2a1$\mathrm{a} 2$, b1-b2). Furthermore, there is no observable surface damage after a single or double pulse action with a pulse energy of $2.5 \mu \mathrm{J}$ (fluence $0.21 \mathrm{~J} \mathrm{~cm}^{-2}$ ) (Fig. 2a1b1). That pulse energy density induces changes after 5 accumulated pulses (Fig. 2c1).

Laser irradiations at pulse energies ranging from 10 to $250 \mu \mathrm{J}$ and the corresponding fluences near and above $1 \mathrm{~J} \mathrm{~cm}^{-2}$, e.g., the "high fluence regime", produce (i) shallow pools of molten material with 5 accumulated pulses of $25 \mu \mathrm{J}$ (Fig. 2c4) and spots induced by pulse energies of 100 and $250 \mu \mathrm{J}$ (Fig. 2a5-a6, b5-b6, c5-c6), (ii) resolidified molten material at the center of the damaged area, even after a single pulse action (Fig. 2a3-a6) and also after a multi-pulse action at 100 and $250 \mu \mathrm{J}$ (Fig. 2b5-b6, c5-c6), (iii) no prominent crater formation and (iv) parallel periodic surface struc- tures at the rims of the irradiated areas (Fig. 2a3-a4, b3-b4).

Further increase of pulse counts $(10,100$ and 400) in the low fluence regime, below $1 \mathrm{~J} \mathrm{~cm}^{-2}$, as expected, induced more pronounced surface features, such as: (i) pool of solidified molten material at the center of the damaged area after 10 accumulated pulses (Fig. 3a1a2), (ii) parallel periodic surface structures at the center of the damaged area, induced by 10 and 100 pulses (Fig. 3a1-a2, b1-b2), (iii) prominent craters induced by 400 accumulated pulses (Fig. 3c1-c2) and (iv) welldefined laser-induced parallel periodic surface structures at the sloping walls of the craters (Fig. 3c1-c2). It can be concluded that incomplete (gentle) ablation, e.g., rearrangement of the material, occurred after laser irradiation with 10 to 100 accumulated pulses, but more efficient removal of the material and therefore effective ablation, resulting in more pronounced craters, occurred after more accumulated pulses.

SEM images of the damaged areas after irradiation with high energy densities, above $1 \mathrm{~J} \mathrm{~cm}^{-2}$ (Fig. 3) and pulse counts from 10 to 400 pulses, show more prominent topographic features: (i) craters at the center of the spot (Fig. 3a4-a6, b3-b6, c3-c6), (ii) spikes of resolidified molten material at the center of the damaged area after 100 accumulated pulses (Fig. 3b3-b4), 

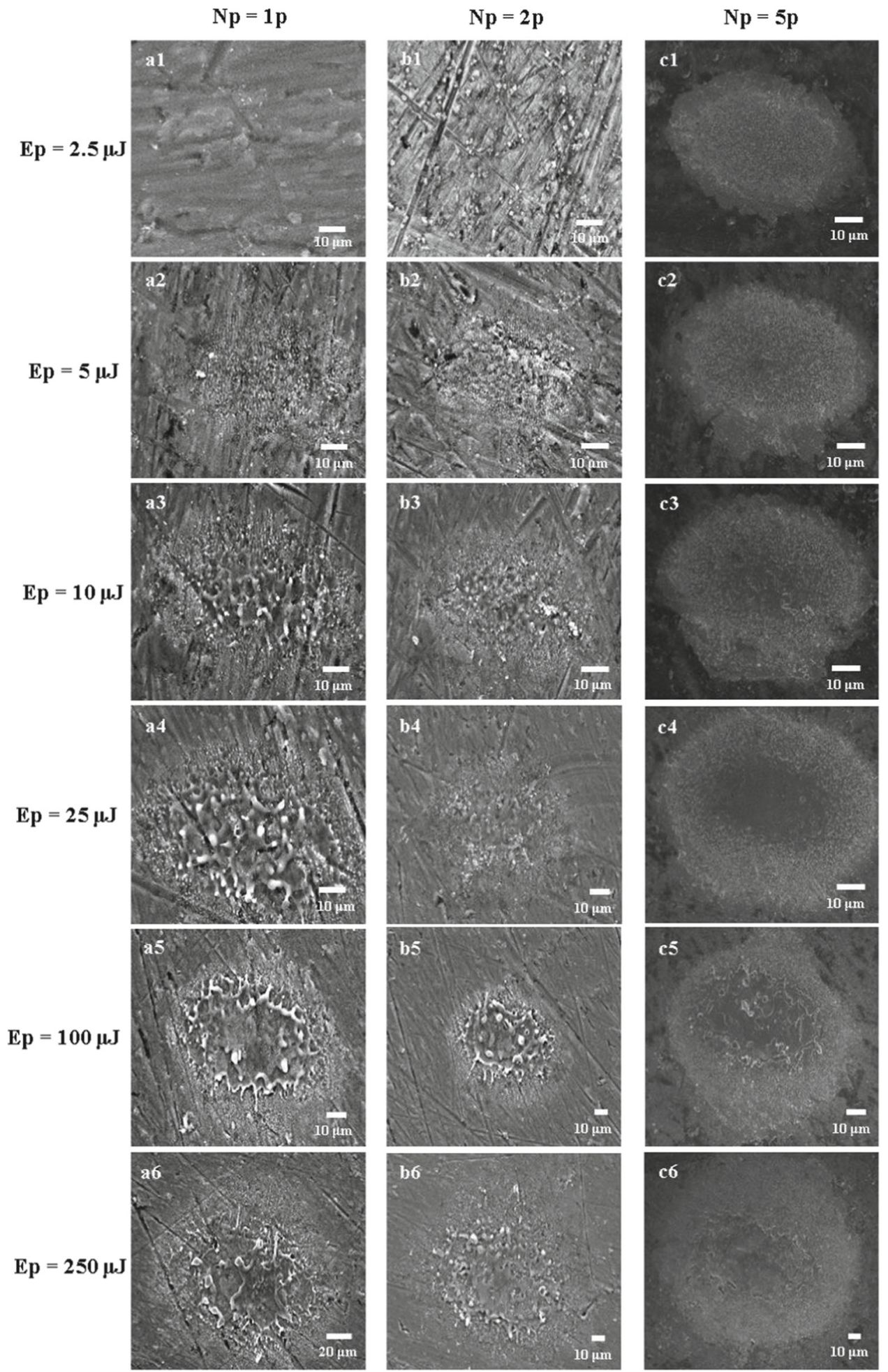

Fig. 2 SEM microphotographs of the Ti6Al4V surface after laser irradiation at 775 nm wavelength, pulse duration 200 fs:

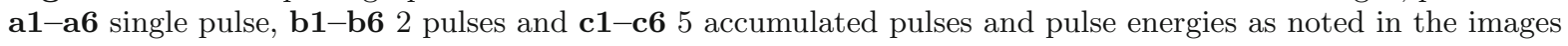



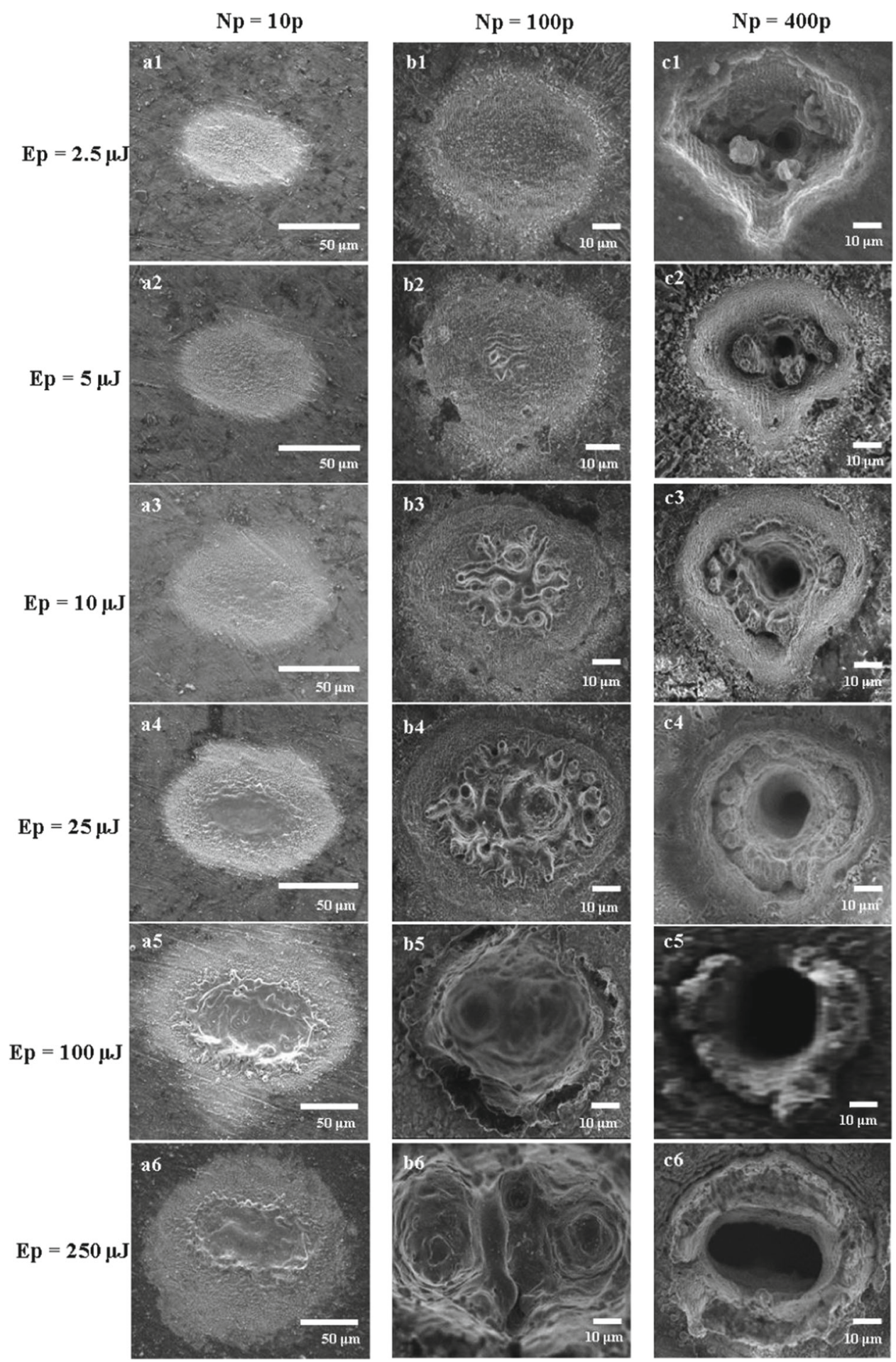

Fig. 3 SEM microphotographs of the Ti6Al4V surface after laser irradiation at 775 nm wavelength, pulse duration 200 fs, pulse count: a1-a6 10 pulses, b1-b6 100 pulses and c1-c6 400 accumulated pulses and pulse energies of $2.5 \mu \mathrm{J}, 5 \mu \mathrm{J}$, $10 \mu \mathrm{J}, 25 \mu \mathrm{J}, 100 \mu \mathrm{J}$ and $250 \mu \mathrm{J}$ 
(iii) resolidified molten material at the periphery and inside the crater (Fig. 3b5-b6, c3-c6). These features imply that ablation and removal of the sample material occurred.

Further characterization was performed by optical profilometry, which provided cross-sectional profiles and three-dimensional maps. Progression of crosssectional profiles of the damaged areas with increasing pulse energy at constant pulse count is presented in Figs. 4 and 5. Dependences of surface parameters, such as average surface roughness, maximum depth and surface area, on the increasing pulse energy and pulse count are presented in Figs. 6, 7 and 8.

The cross-sectional surface profiles after a single pulse irradiation, with energies increasing from 5 to $250 \mu \mathrm{J}$ (Fig. 4a), show the most prominent features of the damaged areas are surface waviness (high fluence regime) or slightly increased surface roughness (low fluence regime). The measurements show that for both fluence regimes, the overall average roughness of $0.2 \mu \mathrm{m}$ (Fig. 8) is close to the average roughness of the untreated surface, $0.18 \mu \mathrm{m}$. At fluences near and above $1 \mathrm{~J} \mathrm{~cm}^{-2}$, after a single pulse irradiation at energies 25 , 100 and $250 \mu \mathrm{J}$, surface waviness $(\sim 1 \mu \mathrm{m})$ occurs.

Following the double pulse action, surface profiles are similar to those after a single pulse irradiations, with an average surface roughness of $0.22 \mu \mathrm{m}$ (Fig. 4b), increased by a factor of 1.2 with respect to untreated surface (Fig. 8). Surface profile evolution after 5 accumulated pulses is shown in Fig. 4c. Formation of shallow craters can be observed after 5 accumulated pulses, at pulse energies 100 and $250 \mu \mathrm{J}$. These results are in agreement with the SEM findings, and the general conclusion is that the 1, 2 and 5 pulses cause gentle ablation.

Profilometry analysis of the damages induced by $10-$ 400 accumulated laser pulses showed that crater formation is the most prominent feature of the irradiated surface (Fig. 5). With 10 accumulated pulses and energies increasing from 2.5 to $25 \mu \mathrm{J}$, only shallow craters of about $\sim 1.5 \mu \mathrm{m}$ depth are formed, as well as increased surface roughness (Fig. 5a). After $10 \mathrm{accu}-$ mulated pulses of 100 and $250 \mu \mathrm{J}$, the craters become more prominent, with increased depth to $\sim 4$ to $5 \mu \mathrm{m}$ (Fig. 5a).

The profiles induced by 50 and 100 accumulated pulses (Fig. 5b, c), respectively, show formation of craters at all pulse energies and increasing of the crater maximum depth with increasing pulse energy, from $2.8 \mu \mathrm{m}$ and $4.93 \mu \mathrm{m}$ at $2.5 \mu \mathrm{J}$, up to $14 \mu \mathrm{m}$ and $33.15 \mu \mathrm{m}$ at $100 \mu \mathrm{J}$, after 50 and 100 laser pulses, respectively. Laser irradiation from 2.5 to $100 \mu \mathrm{J}$ of pulse energy caused efficient removal of the target material and ablation, forming craters with well-defined walls. The expelled molten material, resolidified at the crater rims, is a hydrodynamic effect, caused by melting and rapid cooling of the material between the pulses. Following the laser action of 50 and 100 accumulated pulses of $250 \mu \mathrm{J}$, the craters are characterized by resolidified expelled molten material at the edges of the crater, and spikes of resolidified molten material inside
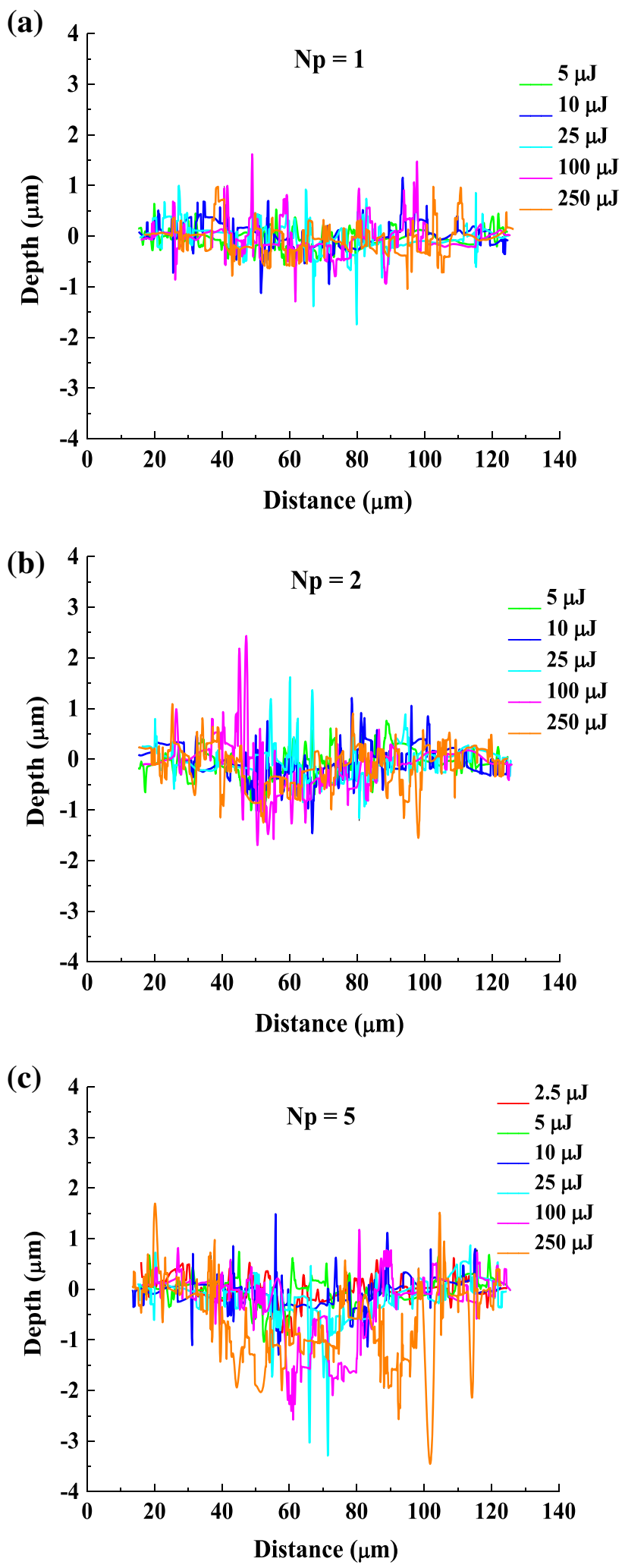

Fig. 4 Cross-sectional profiles of Ti6Al4V surface after laser irradiation at $775 \mathrm{~nm}$ wavelength and pulse duration 200 fs: a single pulse, b 2 pulses and $\mathbf{c} 5$ accumulated pulses. Pulse energies are given in the diagrams 

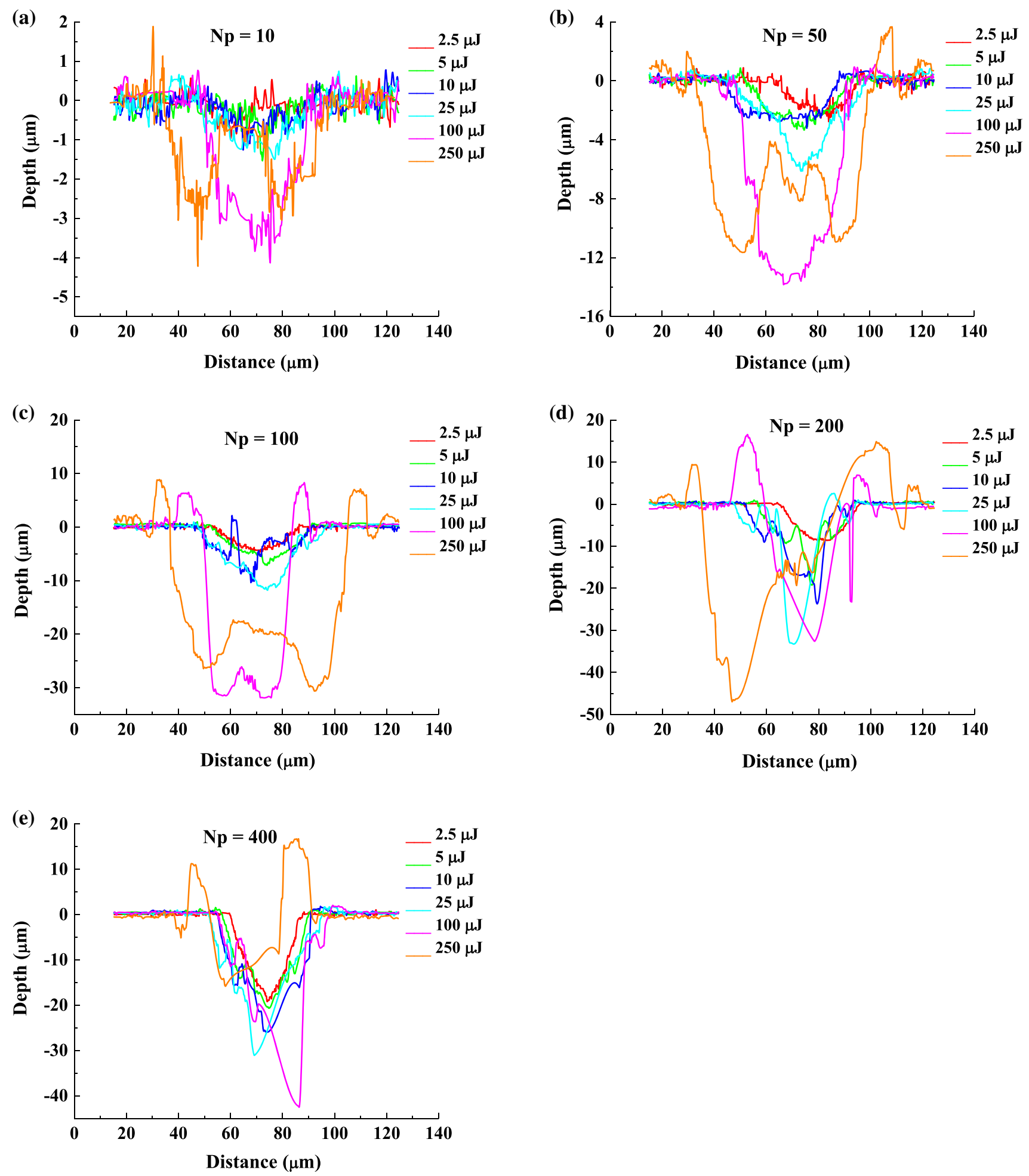

Fig. 5 Cross-sectional profiles of Ti6Al4V surface after laser irradiation at $775 \mathrm{~nm}$ wavelength, pulse duration $200 \mathrm{fs}$, pulse count: a 10 pulses, b 50 pulses, c 100 pulses, d 200 pulses and e 400 accumulated pulses. Pulse energies are given in the diagrams 
the crater, and a consequently slightly decreased maximum depth to $11.8 \mu \mathrm{m}$ and $30.96 \mu \mathrm{m}$ with respect to the effects after 50 and 100 laser pulses of $100 \mu \mathrm{J}$. Some caving-in of crater edges also contributes to this.

Further increase in accumulated pulses also results in crater formation, and the progression of crater dimensions after 200 and 400 accumulated laser pulses is presented in Fig. 5d, e.

Modification of the alloy surface after 200 pulses is characterized by melting and removal of the material (Fig. 5d). A well-defined crater, $9.14 \mu \mathrm{m}$ deep, without hydrodynamic effects at the rims, is formed after $10 \mu \mathrm{J}$ pulses. Additional increase of the pulse count causes increasing maximum depth of the craters up to $47.5 \mu \mathrm{m}$ with $250 \mu \mathrm{J}$ pulses, but with prominent hydrodynamic effects, such as resolidified molten material at the crater rims and spikes of molten material inside the craters, with 100 and $250 \mu \mathrm{J}$ pulses. The overall conclusion is that the ablation led to partial removal of the target material.

Increase of the crater depth from 19.8 to $43 \mu \mathrm{m}$ is also evident after 400 accumulated laser pulses and pulse energies ranging from $2.5 \mu \mathrm{J}$ to $100 \mu \mathrm{m}$ (Fig. 5e). These craters have fairly defined shapes, with sporadic spikes. However, the laser action of 400 accumulated pulses at $250 \mu \mathrm{J}$ caused excessive melting and inefficient removal of the molten material, which can be attributed to caving-in of the edges (the damage spot expands) into the crater, causing a decrease of the crater depth to $16 \mu \mathrm{m}$.

As the pulse energy increases to $10 \mu \mathrm{J}$ and above, melting and subsequent pool of molten material occurs, even with a single laser pulse.

For machining purposes, a fairly clean crater can be achieved within the range of up to $100 \mu \mathrm{J}$ per pulse and not more than 100 accumulated pulses. With lower pulse energies, e.g., $10 \mu \mathrm{J}$, even up to 400 pulses can be used to achieve a controlled effect.

For rough guidance, maximum depths achieved as a function of pulse energy and pulse count are plotted in Fig. 6. The plotted maximum depths are averaged values, with a standard deviation of $5 \%$.

Dependence of the maximum depth on the pulse energy shows that the maximum depth achieved increases with increasing pulse energy, up to 100 accumulated laser pulses and up to $100 \mu \mathrm{J}$ (Fig. 6a). With the further increase of the energy to $250 \mu \mathrm{J}$, the maximum depth at pulse counts from 1 to 100 reaches saturation (Fig. 6a). This inability of deeper drilling changes at the pulse count of 200 , since the maximum depths show a constant increment with pulse energy. With 400 accumulated laser pulses, the maximum depth increases with pulse energy up to $100 \mu \mathrm{J}$, when, instead of further increase or saturation, a decrease is evident (Fig. 6a), showing the inability of removing the molten (and caved-in) material.

Dependence of the maximum depth on the pulse count (Fig. 6b) shows a similar pattern. The maximum depth increases up to 200 pulses, after which point the depth practically decreases, apparently due to redepo-
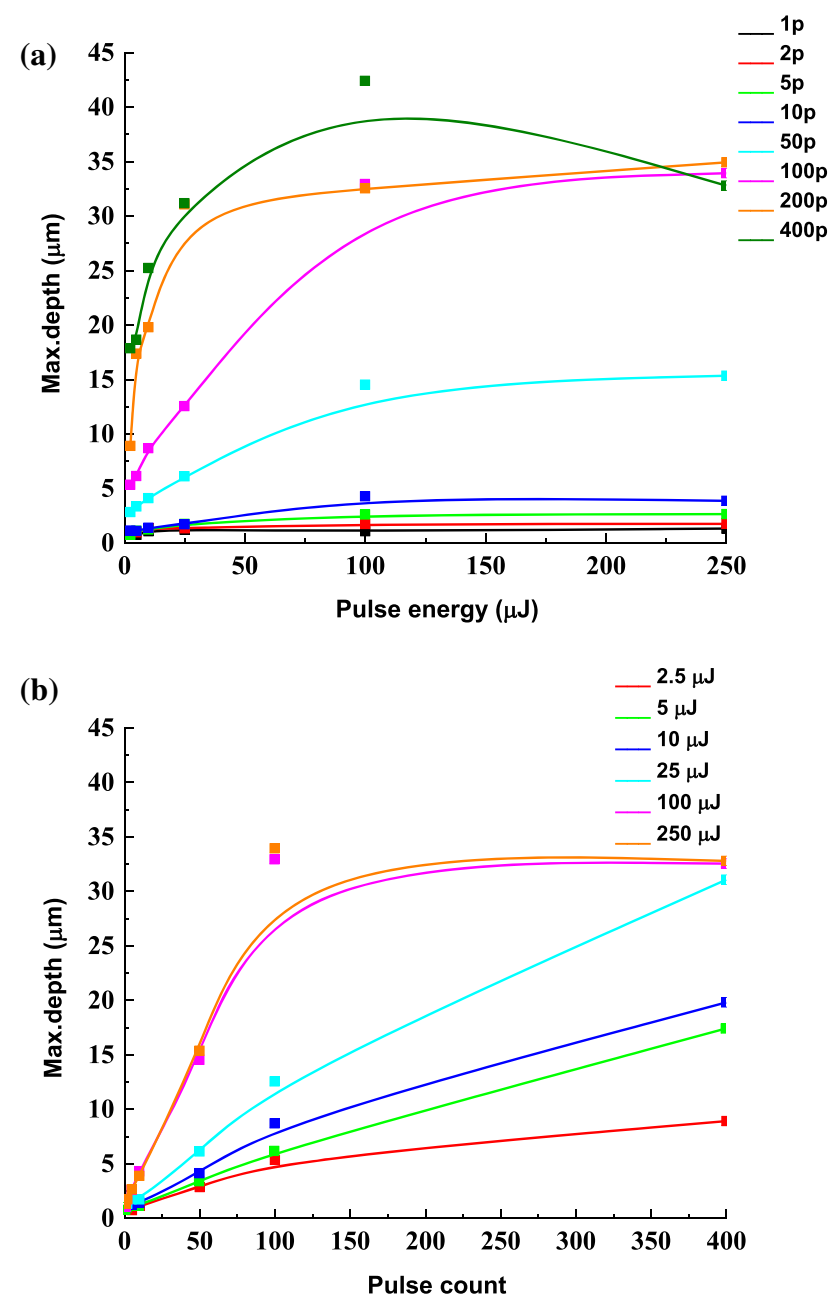

Fig. 6 Maximum depths on the Ti6Al4V surface after laser irradiation at $775 \mathrm{~nm}$ wavelength and pulse duration of $200 \mathrm{fs}$, achieved as a function of a pulse energy ranging from 2.5 to $250 \mu \mathrm{J}$ and $\mathbf{b}$ pulse count ranging from single pulse to 400 accumulated pulses

sition of the material back into the crater and some caving-in of crater sides (Fig. 6b).

Increasing the pulse energy increases the damage spot area (or effective damage diameter), as the energy of the damage threshold in the Gaussian beam moves further out from the center, consequently expanding the crater area. This is visible in Fig. 7 .

Diagram of the dependence of the average surface roughness of the treated area with increasing number of accumulated pulses is shown in Fig. 8. It is evident that the present femtosecond laser action increases the roughness to an average of $3.8 \mu \mathrm{m}$ after 400 accumulated pulses.

There are studies proposing that the regions of low and high fluence are defined by optical penetration depth and electronic heat conduction, subsequently $[31,32]$. These studies show these regions may be determined by intercrossing of the linear fits of the ablation rate, i.e., maximal ablation depth per pulse, as a function of laser fluence for $80 \mathrm{fs}$ laser pulse, $800 \mathrm{~nm}$ 


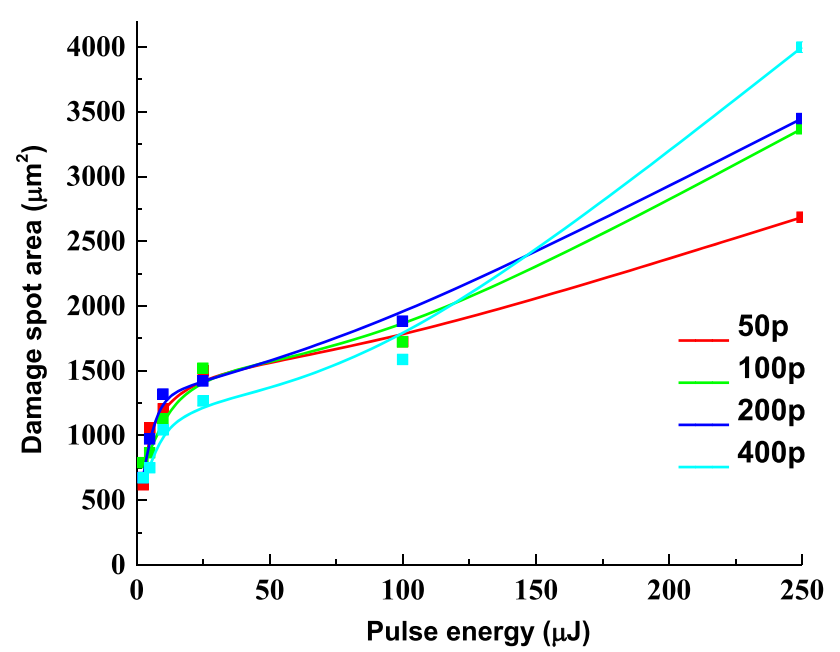

Fig. 7 Dependence of damage spot area of the Ti6Al4V surface, after laser irradiation at $775 \mathrm{~nm}$ wavelength and pulse duration of $200 \mathrm{fs}$, on the pulse energies from 2.5 to 250 $\mu \mathrm{J}$, with pulse count ranging from 50 to 400 accumulated pulses

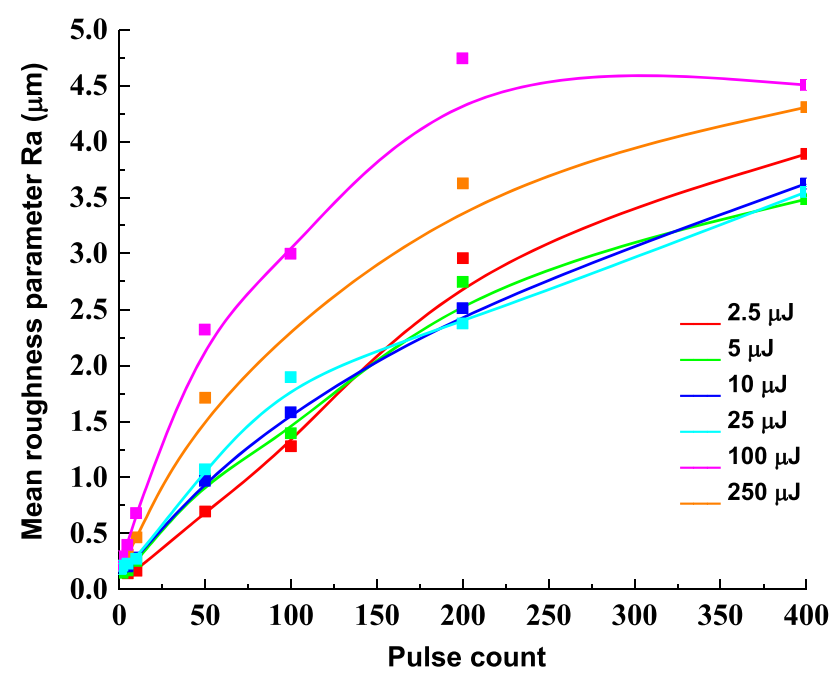

Fig. 8 Average surface roughness achieved on the Ti6Al4V surface, after laser irradiation at $775 \mathrm{~nm}$ wavelength and pulse duration $200 \mathrm{fs}$, as a function of pulse count, from single pulse to 400 accumulated pulses, at pulse energies from 2.5 to $250 \mu \mathrm{J}$

wavelength, $1 \mathrm{~mJ}$ maximum energy and $1 \mathrm{kHz}$ repetition rate when the target is titanium and the modification is done in vacuum [31] or that the fluence of 1 $\mathrm{J} \mathrm{cm}^{-2}$ is considered the distinctive value between low and high fluence regime for cleaning of Ti6Al4V by laser action of 100 pulses, 130 fs pulse duration, at $790 \mathrm{~nm}$ wavelength and repetition rate of $1 \mathrm{KHz}$ [32]. However, in the present case, with increased pulse count and a repetition rate to $2 \mathrm{~Hz}$, as well as a different level of alloy surface preparation, the femtosecond laser action causes pronounced surface features, like craters, even when the applied laser fluence value may be labelled as "low". This is the reason we used the terms "low flu- ence regime" and "high fluence regime", regarding not only laser fluence, but also pulse count.

More detailed SEM analyses of irradiated areas revealed the occurrence of periodic surface structures (Figs. 9, 10 and 11), and our findings are briefly discussed below.

The mechanisms of LIPSS formation have been investigated and reported, but the theory of their origin is still an ongoing research. These LIPSS can be a result of the interference of an incoming laser beam with a surface scattered wave [23]. These waves, in the case of metals, are caused by the coupling of electrons with photons causing resonant oscillations which result in the propagation of a surface wave. Fundamentally, LIPSS are complex as their formation includes a "mashup" of thermal and possibly hydrodynamic or chemical effects. Parallel and net-like laserinduced periodic surface structures (LIPSS) can be observed at a pulse energy of $2.5 \mu \mathrm{J}$ (Fig. 9). After 5 accumulated pulses, net-shaped structures with periods $\sim 670 \mu \mathrm{m}$ can be observed at the center of the spot (Fig. 9a1). More defined, parallel structures are observed at the periphery of the spot (Fig. 9a2). The periods of these surface structures decrease to $150 \mathrm{~nm}$ toward the periphery of the spot. These structures evolve into periodic parallel ripples after 10 accumulated laser pulses, at the same pulse energy (Fig. 9b1b2). Periodic structures in the form of parallel ripples are observable across the whole spot area affected by the laser beam, with an average period of $\sim 700 \mathrm{~nm}$ at the center (Fig. 9b1) and $\sim 570 \mathrm{~nm}$ at the periphery (Fig. 9b2). Low-frequency parallel periodic surface structures (LF-LIPSS) [23] are dependent on the laser beam wavelength and beam polarization and their theoretical period is approximately $775 \mathrm{~nm}$, using the equation $\tau \approx \frac{\lambda_{\text {laser }}}{\cos \left[\Theta_{i}\right]}[33]$, where $\lambda_{\text {laser }}$ is the laser wavelength and $\Theta_{i}$ is the laser beam incident angle. Evidently, the $E$ vector is perpendicular to ripples - type I (long period-from 400 to $700 \mathrm{~nm}$ ), e.g. polarization with horizontal direction (Figs. 9, 10 and 11). The p or s-polarization has no meaning in this context because the laser irradiation was with normal incidence, so the equation $\tau \approx \frac{\lambda_{\text {laser }}}{\cos \left[\Theta_{i}\right]}[33]$ is used only for estimation of the expected LIPSS period.

Appearance of laser-induced parallel periodic surface structures is observable on the Ti6Al4V surface even after the single and double pulse treatments, as well as at the increased pulse energy of $5 \mu \mathrm{J}$ (Fig. 10a1-a2). It is noticed that the periods also decrease toward the periphery of the modified area, from $710 \mathrm{~nm}$ average period at the center (Fig. 10a1-b1) to $530 \mathrm{~nm}$ periods at the periphery (Fig. 10a2-b2).

With increasing pulse energy and pulse count, the ripples are formed with all laser energies used, albeit at the periphery of the spot in cases of higher pulse energies (Fig. 11). The periods also decrease with pulse count so that the average period after 100 pulses is $\sim 400 \mathrm{~nm}$, thus creating high-frequency ripples, HFLIPSS [23] with a period of approximately $60 \%$ of $\lambda$ (laser wavelength) (Fig. 11a2). In cases when craters 

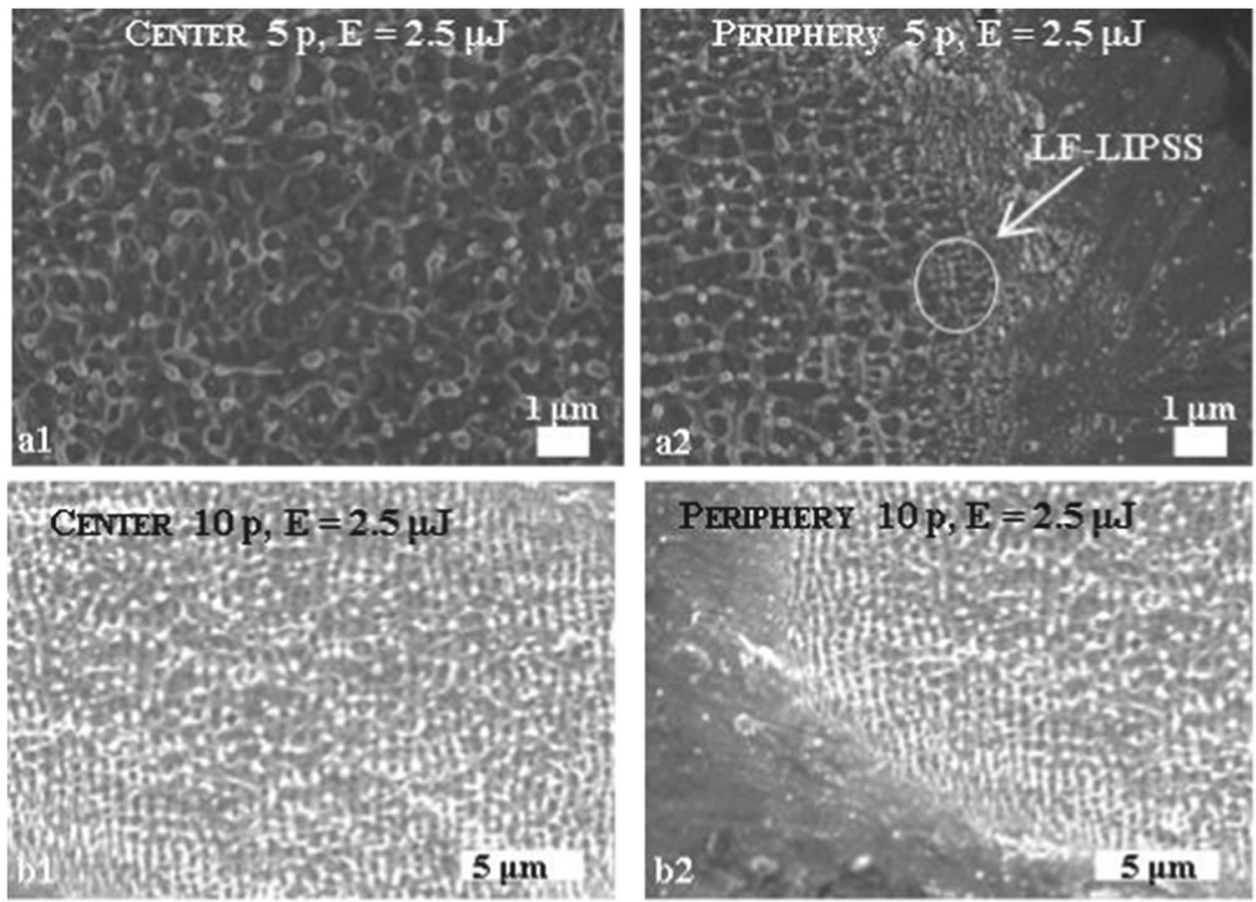

Fig. 9 Periodic surface structures formed on the Ti6Al4V surface, after laser irradiation at $775 \mathrm{~nm}$ wavelength, $200 \mathrm{fs}$ pulse duration and $2.5 \mu \mathrm{J}$ pulse energy, at the center and periphery of the damage spots: a1 center and a2 periphery after 5 pulses; and $\mathbf{b} \mathbf{1}$ center and $\mathbf{b} \mathbf{2}$ periphery after 10 accumulated pulses
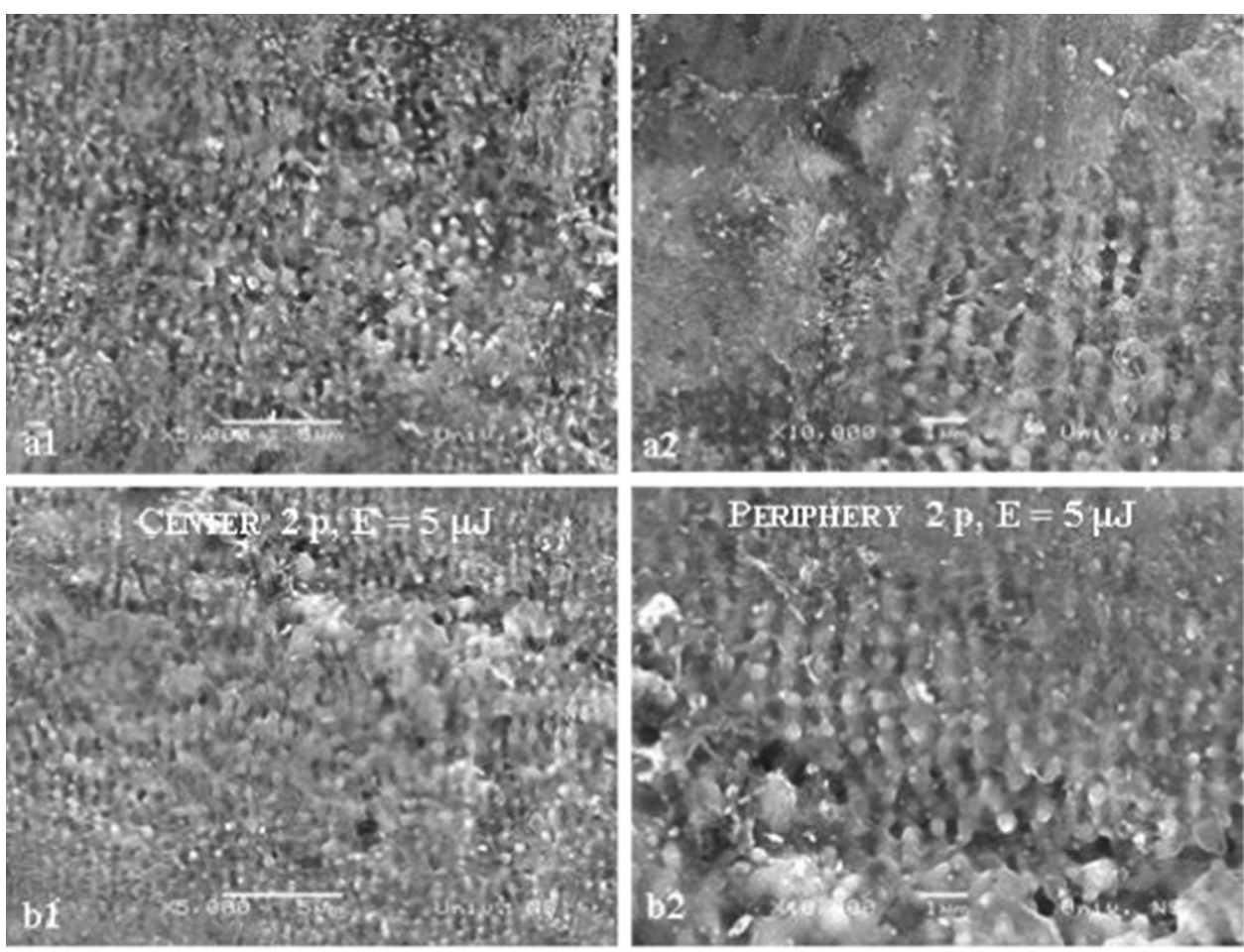

Fig. 10 Periodic surface structures formed on the Ti6Al4V surface after laser irradiation at $775 \mathrm{~nm}$ wavelength, $200 \mathrm{fs}$ pulse duration and $5 \mu \mathrm{J}$ pulse energy, at the center and periphery of the damage spot areas: a1 center and a2 periphery after a single laser pulse; and b1 center and b2 periphery after 2 pulses 

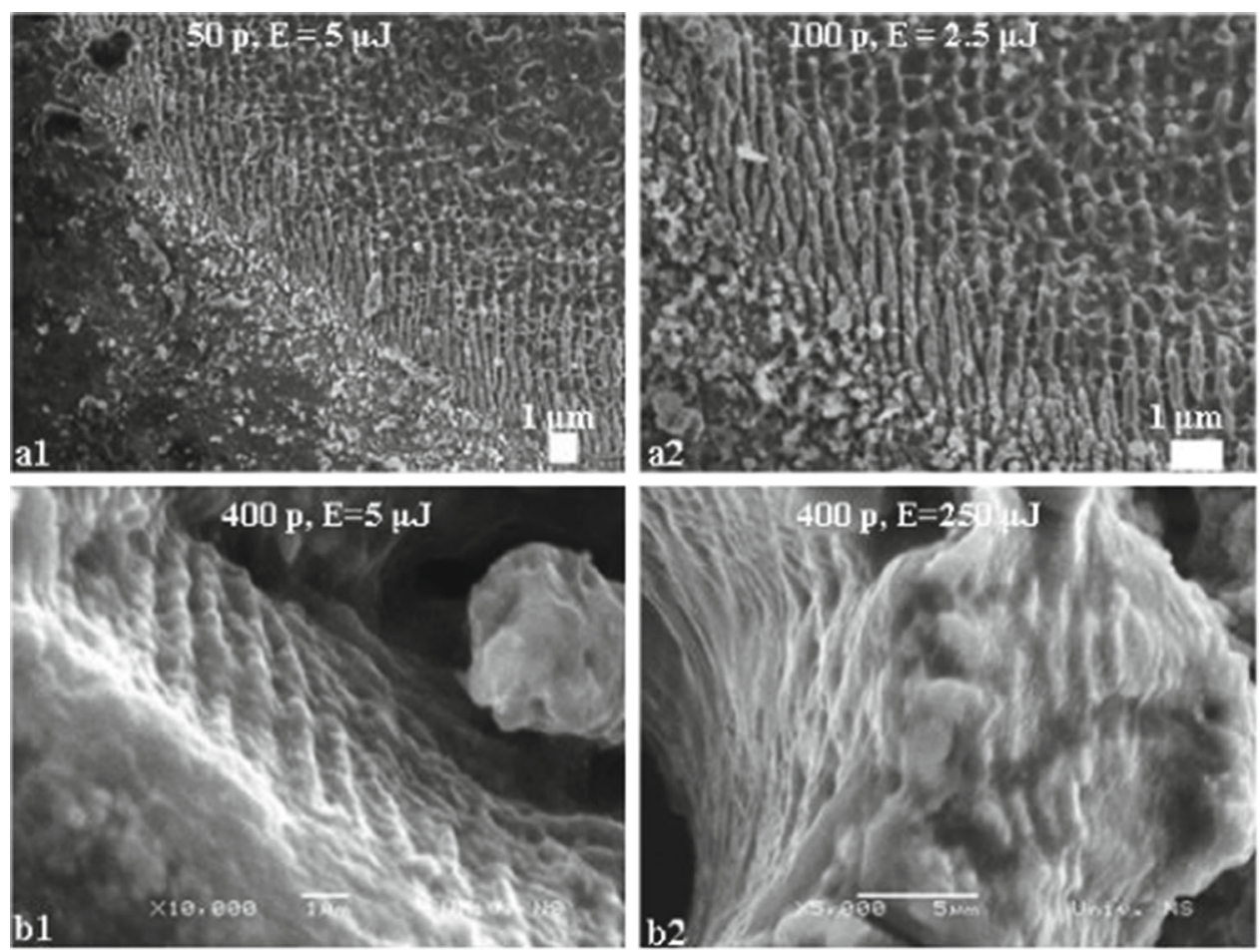

Fig. 11 Periodic surface structures formed on the Ti6Al4V surface, after laser irradiation at $775 \mathrm{~nm}$ wavelength, $200 \mathrm{fs}$ pulse duration: a1 50 pulses of $5 \mu \mathrm{J}, \mathbf{b} 100$ pulses of $2.5 \mu \mathrm{J}, \mathbf{b} 1400$ pulses of $5 \mu \mathrm{J}$ and b2 400 pulses of $250 \mu \mathrm{J}$

are formed, an interesting feature is the formation of ripples even at crater walls (Fig. 11b1). Additionally, the ripples can be observed on the spikes of expelled solidified molten material (Fig. 11b2).

LIPSS structures resulting from femtosecond laser with laser wavelength $\sim 1030 \mathrm{~nm}$ with fluence between 0.16 and $1 \mathrm{~J} \mathrm{~cm}^{-2}$ show periods of $720-860 \mathrm{~nm}$ for these structures [34-37], while femtosecond laser with laser wavelength of $\sim 790 \mathrm{~nm}$ an fluence of $0.11 \mathrm{~J} \mathrm{~cm}^{-2}$ shows LIPSS structures with a period of $\sim 600 \mathrm{~nm}$ [38]. Considering periods of LIPSS emerging from this work (530-700 nm), there is a good agreement with results of other authors and literature; periods of LIPSS structures are below the laser wavelength, around 0.75 $\lambda_{\text {wavelength }}$. The observed LIPSS periods are not significantly affected by surface deformations/irregularities.

Important data for laser processing of this alloy are the minimal energy density sufficient to cause irreversible surface damage, known as the threshold fluence, $F_{\mathrm{th}}$. The calculation of the threshold fluence and laser beam diameter is based on the equation $D^{2}=$ $2 \omega_{0}^{2} \cdot \ln \frac{F_{0}}{F_{\mathrm{th}}}$, where D represents the diameter of the modified area, $\omega_{0}$ laser beam diameter, $F_{o}$ peak fluence, $F_{\text {th }}$ is the threshold fluence, and the linear dependence of the peak fluence and the corresponding output laser energy, $E_{p}: F_{0}=\frac{2 \cdot E_{\mathrm{p}}}{\pi \cdot \omega_{0}^{2}}[30]$. The procedure includes plotting of the damage diameters on a semilogarithmic scale [29]. Most metallic materials exhibit a decrease in damage threshold with increase of the pulse count [39]. Fig. 12a shows a semilogarithmic plot for 1, 2, 5, 10, 100 and 400 accumulated pulses. Threshold fluence,
$F_{\text {th }}$, was determined from the slope and the intercept values of the linear fit (Fig. 12a). Laser beam diameter $\omega_{0}$ determined from the slope of the single pulse linear is $26.8 \mu \mathrm{m}$.

Due to the accumulation effects, the threshold fluence value changes with increasing pulse counts, as presented in Table 2, and the diagram in Fig. 12b, the threshold fluence significantly decreases after the first 10 pulses. Considering the trend in Fig. 12b, it can be concluded that this decrease is not indefinite, and that after 400 accumulated pulses, it probably reaches saturation. The decrease of the $F_{\text {th }}$ by increase of the pulse count can be explained by the change in reflection after the first few pulses, which causes higher absorbance of the laser energy by the target, and subsequently, higher ablation rate and the increase of the energy coupling mechanism between target and the laser beam due to the excitation of surface plasmons [32]. The overall decrease from the single pulse damage threshold fluence to 400 pulses damage threshold fluence is by a factor of 4.5.

In the single pulse irradiation, threshold fluence is mostly dependent on the material thermal properties. In the present experiment, the value of $0.441 \mathrm{~J} \mathrm{~cm}^{-2}$ is higher than the energy density value of $0.22 \mathrm{~J} \mathrm{~cm}^{-2}$ corresponding to the $2.5 \mu \mathrm{J}$ energy, Table 1 , for the single pulse ablation. This is the reason why no surface damage could be observed after 1 single pulse action at $2.5 \mu \mathrm{J}$ pulse energy. With accumulating laser pulses, the incubation effects lead to increasing efficiency in energy coupling [39] causing a decrease of threshold damage (Table 2), and the modification of the surface occurs 

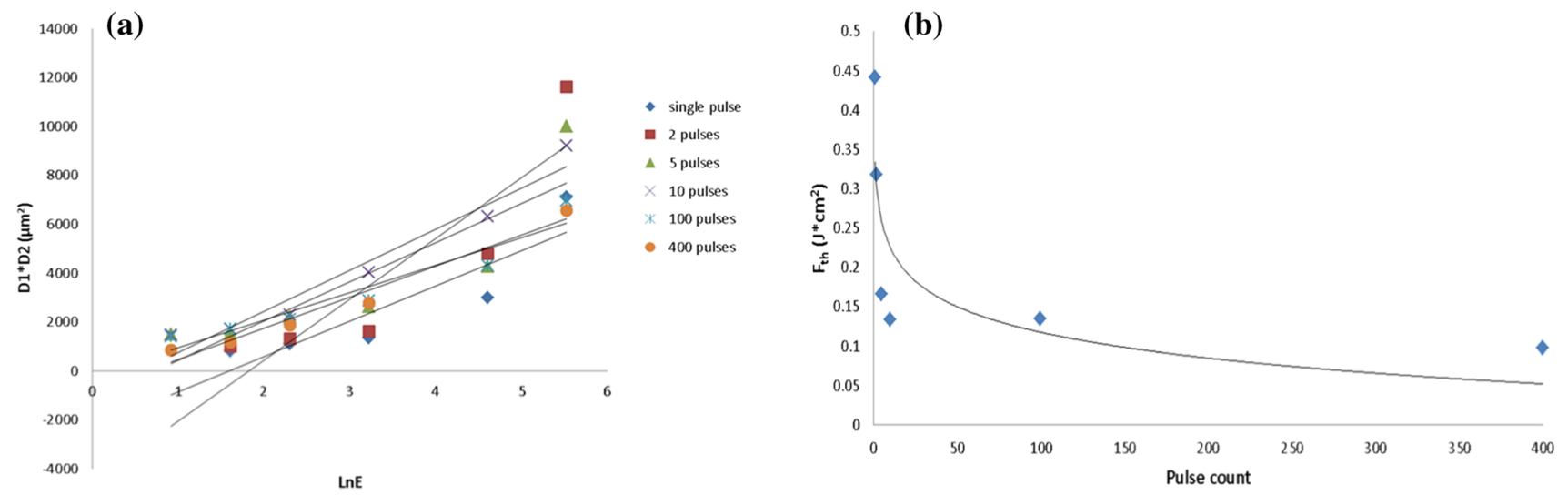

Fig. 12 a Semilogarithmic plot of $D^{2}$ for Ti6Al4V and b dependence of threshold fluence on increasing pulse count

Table 2 Damage threshold fluence at different pulse counts

\begin{tabular}{lllllll}
\hline Pulse count & 1 & 2 & 5 & 10 & 100 & 400 \\
\hline Threshold fluence, $F_{\mathrm{th}}\left(\mathrm{J} \mathrm{cm}^{-2}\right)$ & 0.441 & 0.317 & 0.165 & 0.133 & 0.134 & 0.097 \\
\hline
\end{tabular}

due to the multi-pulse irradiation at $2.5 \mu \mathrm{J}$ laser energy. The decrease of the threshold fluence with accumulated laser pulses is in agreement with previous studies [32].

For the presented laser/material interaction, the degree of incubation is determined following the incubation model [30]. The dependence of the damage threshold fluence for $\mathrm{N}$ pulses, $F_{\mathrm{th}}(N)$, and damage threshold fluence for 1 pulse, $F_{\mathrm{th}}(1)$, is described by the equation $\mathrm{F}_{\mathrm{th}}(\mathrm{N})=\mathrm{F}_{\mathrm{th}}(1) \cdot \mathrm{N}^{\zeta-1}$, where $\xi$ is the incubation factor which characterizes the response of the target material to the multi-pulse ablation, i.e., degree of incubation $[22,30]$. The incubation factor $\zeta$ determined from the slope of the linear fit of the dependence $\log \left(N \cdot F_{\text {th }}(N)\right)=f(\log N)$ (Fig. 13) has the value of 0.78 for Ti6Al4V at the present experimental conditions.

The incubation model was also used to determine/ confirm the damage threshold fluence for single pulse ablation $F_{\mathrm{th}}(1)$, from the intercept of the linear fit (Fig. 13). The value of $0.49 \mathrm{~J} \mathrm{~cm}^{-2}$ for $F_{\mathrm{th}}(1)$ is in good agreement with the value of $0.44 \mathrm{~J} \mathrm{~cm}^{-2}$, Table 2 . Previous studies reported incubation factor of 0.83 and $F_{\mathrm{th}}(1)$ of $0.28 \mathrm{~J} \mathrm{~cm}^{-2}$ for titanium [30] and 0.855 and $0.27 \mathrm{~J} \mathrm{~cm}^{-2}$ for Ti6Al4V [32]. Our results differ from these studies probably due to the higher value of repetition rate (by a factor of 2) and more surface defects/irregularities which might have prevented the observation of the effects at low values of peak laser energy.

\section{Conclusions}

- The surface of the titanium-based alloy Ti6Al4V was subjected to modifications by a near-IR femtosecond Ti:Sapphire laser, emitting at $775 \mathrm{~nm}$

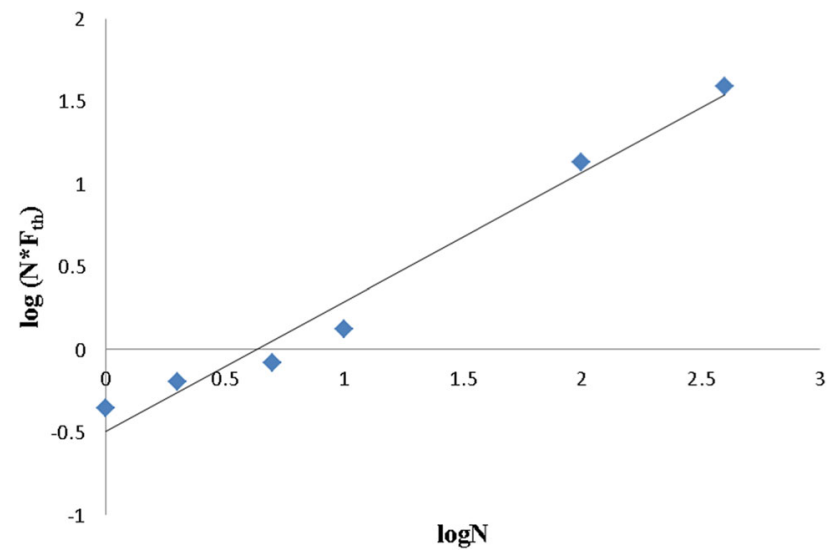

Fig. 13 a Logarithmic plot and linear fit of the dependence $\log \left(N \cdot F_{\text {th }}(N)\right)=f(\log N)$, characteristic for multipulse interaction of 200 fs laser beam with Ti6Al4V alloy surface

pulses of 200 fs duration, in single-pulse and multipulse regimes, with up to 400 accumulated pulses, and pulse energies ranging from $2.5 \mu \mathrm{J}$ to $250 \mu \mathrm{J}$.

- The resulting effects at a low fluence regime (significantly below $1 \mathrm{~J} \mathrm{~cm}^{-2}$ ) and 1, 2 or 5 accumulated pulses can be described as gentle ablation. Irradiation with the same pulse counts at a high fluence regime (near and above $1 \mathrm{~J} \mathrm{~cm}^{2}$ ) caused more pronounced surface features, but no efficient removal of material.

- Increasing the pulse counts to 400 at low fluence regime induced gentle ablation, e.g., rearrangement of the material occurred after 10-100 accumulated pulses, but more efficient removal of the material, and therefore effective ablation, resulting in more pronounced craters, occurred at higher pulse counts. 
- After irradiation at the high fluence regime and pulse counts from 10-400, ablation with removal of the sample material occurred.

- Average surface roughness increased with the increasing pulse energy and number of accumulated pulses by a factor of $\sim 20$.

- Laser-induced parallel periodic surface structures are observable with periods ranging from $710 \mathrm{~nm}$ to as low $150 \mathrm{~nm}$. Such structures are apparent even after single and double pulse treatments with pulse energies of $5 \mu \mathrm{J}$.

- Damage threshold fluence for a single pulse irradiation is found to be $0.441 \mathrm{~J} \mathrm{~cm}^{-2}$, its value decreasing to $0.097 \mathrm{~J} \mathrm{~cm}^{-2}$ for 400 accumulated pulses.

- The incubation factor is determined to be 0.78 for this alloy at the presented experimental conditions.

- Generally, as the pulse energy increases to $10 \mu \mathrm{J}$ and above, melting and subsequent pool of molten material occurs, even with a single laser pulse.

- For machining purposes, a fairly clean crater can be achieved within the range of up to $100 \mu \mathrm{J}$ per pulse, and not more than 100 accumulated pulses. With lower pulse energies, e.g., $10 \mu \mathrm{J}$, even up to 400 pulses can be used to achieve a controlled effect.

- The results show that using a moderate optical quality sample surface and a repetition rate of $2 \mathrm{kHz}$ and several hundred pulses, a femtosecond laser can produce pronounced surface features at lower fluences than with a high quality surface. This finding can have a practical value too.

- All results point to the fact that more research along these lines can be beneficial, since the outcome of laser processing of Ti6Al4V can depend on several parameters, like laser beam characteristics, repetition rate, surface quality, number of accumulated pulses in combination with fluence, etc.

Acknowledgements The research was sponsored by the Ministry of Education, Science and Technological Development of the Republic of Serbia through contracts Nos. 45103-9/2021-14/200051 and 451-03-9/2021-14/200017 and by the Romanian Ministry of Education and Research, under Romanian National Nuclei Program LAPLAS VI - contract no. $16 \mathrm{~N} / 2019$. We also acknowledge the support from European Community, COST Action CA17126.

\section{Author contributions}

DM and BR conceived and planned the experiments. $\mathrm{BR}, \mathrm{DM}, \mathrm{MZ}, \mathrm{CA}$ and $\mathrm{AR}$ carried out the experiments. All authors contributed to the interpretation of the results. DM took the lead in writing the manuscript. BR, BG and JS provided critical feedback and helped shape the analysis and manuscript.

Data Availability Statement This manuscript has no associated data or the data will not be deposited. [Authors' comment: All relevant data is included in the paper.]
Open Access This article is licensed under a Creative Commons Attribution 4.0 International License, which permits use, sharing, adaptation, distribution and reproduction in any medium or format, as long as you give appropriate credit to the original author(s) and the source, provide a link to the Creative Commons licence, and indicate if changes were made. The images or other third party material in this article are included in the article's Creative Commons licence, unless indicated otherwise in a credit line to the material. If material is not included in the article's Creative Commons licence and your intended use is not permitted by statutory regulation or exceeds the permitted use, you will need to obtain permission directly from the copyright holder. To view a copy of this licence, visit http://creativecomm ons.org/licenses/by/4.0/.

\section{References}

1. W.S. Lee, C.F. Lin, Plastic deformation and fracture behaviour of Ti-6Al-4Valloy loaded with high strain rate under various temperatures. Mater. Sci. Eng. A 241, 48-59 (1998)

2. J. Parthasarathy, B. Starly, S. Raman, A. Christensen, Mechanical evaluation of porous titanium (Ti6Al4V) structures with electron beam melting (EBM). J. Mech. Behav. Biomed. Mater. 3, 249-259 (2010)

3. J. Xu, W. Zeng, X. Sun, Z. Jia, Microstructure evolution during isothermal forging and subsequent heat treatment of Ti-17 alloy with a lamellar colony structure. J. Alloys Compd. 637, 449-455 (2015)

4. Q. Zhang, Z.L. Liang, M. Cao, Z.F. Liu, A.F. Zhang, B.H. Lu, Microstructure and mechanical properties of Ti6Al4V alloy prepared by selective laser melting combined with precision forging. Trans. Nonferrous Met. Soc. China 27, 1036-1042 (2017)

5. Y. Zhu, D. Liu, X. Tian, H. Tang, H. Wang, Characterization of microstructure and mechanical properties of laser melting deposited $\mathrm{Ti}-6.5 \mathrm{Al}-3.5 \mathrm{Mo}-1.5 \mathrm{Zr}-0.3 \mathrm{Si}$ titanium alloy. Mater. Des. 56, 445-453 (2014)

6. X. Liu, P.K. Chu, C. Ding, Surface modification of titanium, titanium alloys, and related materials for biomedical applications. Mater. Sci. Eng. R 47, 49-121 (2004)

7. M. Peters, J. Kumpfert, C.H. Ward, C. Leyens, Titanium Alloys for Aerospace Applications. Adv. Eng. Mater. 5, 419-427 (2003)

8. A.S. Oryshchenko, I.V. Gorynin, V.P. Leonov, A.S. Kudryavstev, V.I. Mikhailov, E.V. Chudakov, Marine titanium alloys: present and future. Inorg. Mater. Appl. Res. 6, 571-579 (2015)

9. L.C. Zhang, L.Y. Chen, L. Wang, Surface modification of titanium and titanium alloys: technologies, developments, and future interests. Adv. Eng. Mater. 22, 1901258 (2020)

10. O. Adesina, P. Popoola, O. Fatoba, Laser surface modification - a focus on the wear degradation of titanium alloy, in Fiber Laser. ed. by M.C. Paul (IntechOpen, London, 2016)

11. L.C. Zhang, H. Attar, Selective laser melting of titanium alloys and titanium matrix composites for biomedical applications: a review. Adv. Eng. Mater. 18, 463-475 (2015) 
12. O. Raimbault, S. Benayoun, K. Anselme, C. Mauclair, T. Bourgade, A.M. Kietzig, P.L. Girard-Lauriault, S. Valette, C. Donnet, The effects of femtosecond lasertextured Ti-6Al-4V on wettability and cell response. Mater. Sci. Eng. C 69, 311-320 (2016)

13. S. Yadav, A. Kumar, T. Paramesh, K. Sunita, A review on enhancement of wear resistance properties of titanium alloy using nano composite coating. Mater. Sci. Eng. 455, 012120 (2018)

14. M. Geetha, A.K. Singh, R. Asokamani, A.K. Gogia, Ti based biomaterials, the ultimate choice for orthopaedic implants - a review. Prog. Mater. Sci. 54, 397-425 (2009)

15. L.H. Liu, C. Yang, F. Wang, S.G. Qu, X.Q. Li, W.W. Zhang, Y.Y. Li, L.C. Zhang, Ultrafine grained Tibased composites with ultrahigh strength and ductility achieved by equiaxing microstructure. Mater. Des. 79, 1-5 (2015)

16. M. Niinomi, Fatigue performance and cyto-toxicity of low rigidity titanium alloy, Ti-29Nb-13Ta-4.6Zr. Biomaterials 24, 2673-2683 (2003)

17. L.C. Zhang, L.Y. Chen, A review on biomedical titanium alloys: recent progress and prospect. Adv. Eng. Mater. 21, 1801215 (2019)

18. C. Zwarh, R. Helbig, C. Werner, A.F. Lasagni, Fabrication of multifunctional titanium surfaces by producing hierarchical surface patterns using laser based ablation methods. Sci. Rep. 9, 6721 (2019)

19. A. Cunha, V. Oliveira, R. Vilar, Ultrafast laser surface texturing of titanium alloys. Lasers Surf. Modif. Biomater. 56, 301-322 (2016)

20. D.D. Lima, S.A. Mantri, C.V. Mikler, R. Contieri, C.J. Yannetta, K.N. Campo, E.S. Lopes, M.J. Styles, T. Borkar, R. Caram, R. Banerjee, Laser additive processing of a functionally graded internal fracture fixation plate. Mater. Des. 130, 8-15 (2017)

21. M. Huang, F. Zhao, Y. Cheng, N. Xu, Z. Xu, Origin of laser-induced near-subwavelength ripples: interference between surface plasmons and incident laser. ACS Nano 3, 4062-4070 (2009)

22. B. Gakovic, C. Radu, M. Zamfirescu, B. Radak, M. Trtica, S. Petrovic, P. Panjan, F. Zupanic, C. Ristoscu, I.N. Mihailescu, Femtosecond laser modification of multilayered TiAlN/TiN coating. Surf. Coat. Technol. 206, 411-416 (2011)

23. J. Bonse, S. Hohm, S.V. Kirner, A. Rosenfeld, J. Kruger, Laser-induced periodic surface structures - a scientific evergreen. IEEE J. Sel. Top. Quantum Electron. 23, 115 (2017)

24. L. Mellor, S.P. Edwardson, W. Perrie, G. Dearden, K. Watkins, Ultrashort pulse periodic surface structuring of metallic surfaces, J. Laser Appl., International Congress on Applications of Lasers \& Electro-Optics 2010, pp. 791-798 (2010)

25. J.E. Sipe, J.F. Young, J.S. Preston, H.M. van Driel, Laser-induced periodic surface structure. I. Theory. Phys. Rev. B 27, 1141-1154 (1983)

26. J.F. Young, J.S. Preston, H.M. van Driel, J.E. Sipe, Laser-induced periodic surface structure. II. Experiments on Ge, Si, Al, and brass. Phys. Rev. B 27, 1155$1172(1983)$

27. J.F. Young, J.E. Sipe, H.M. van Driel, Laser-induced periodic surface structure. III. Fluence regimes, the role of feedback, and details of the induced topography in germanium. Phys. Rev. B 30, 2001-2015 (1984)

28. A. Cimpean, V. Mitran, C.M. Ciofrangeanu, B. Galateanu, E. Bertrand, D.M. Gordin, D. Iordachescu, T. Gloriant, Osteoblast cell behavior on the new betatype Ti-25Ta-25Nb alloy. Mater. Sci. Eng. C 32, 1554$1563(2012)$

29. N.M. Bulgakova, I.M. Burakov, Y.P. Meshcheryakov, R. Stoian, A. Rosenfeld, I.V. Hertel, Theoretical models and qualitative interpretations of Fs laser material processing. J. Laser Micro Nanoeng. 2, 76-86 (2007)

30. P.T. Mannion, J. Magee, E. Coyne, G.M. O'Connor, T.J. Glynn, The effect of damage accumulation behaviour on ablation thresholds and damage morphology in ultrafast laser micro-machining of common metals in air. Appl. Sur. Sci. 233, 275-287 (2004)

31. M. Ye, C.P. Grigoropoulos, Time-of-flight and emission spectroscopy study of femtosecond laser ablation of titanium. J. Appl. Phys. 89, 5183-5190 (2001)

32. N. Maharjan, W. Zhou, Y. Zhou, Y. Guan, Ablation morphology and ablation threshold of Ti-6Al-4V alloy during femtosecond laser processing. Appl. Phys. A 124, 519 (2018)

33. K.K. Venkatakrishnan, B. Tan, Interconnect microvia drilling with a radially polarized laser beam. J. Micromech. Microeng. 16, 2603 (2006)

34. G. Rotella, L. Orazi, M. Alfano, S. Candamano, I. Gnilitskyi, Innovative high-speed femtosecond laser nanopatterning for improved adhesive bonding of Ti6Al4V titanium alloy. CIRP J. Manuf. Sci. Technol. 18, 101106 (2017)

35. I. Gnilitskyi, M. Pogorielov, R. Viter, A.M. Ferraria, A.P. Carapeto, O. Oleshko, L. Orazi, O. Mishchenko, Cell and tissue response to nanotextured Ti6Al4V and Zr implants using high-speed femtosecond laser-induced periodic surface structures. Nanomedicine 21, 102036 (2019)

36. D. Huerta-Murillo, A.I. Aguilar-Morales, S. Alamri, J.T. Cardoso, R. Jagdheesh, A.F. Lasagni, J.L. Ocana, Opt. Lasers Eng. 98, 134-142 (2017)

37. D. Huerta-Murillo, A. Garcia-Giron, J.M. Romano, J.T. Cardoso, F. Cordovilla, M. Walker, S.S. Dimov, J.L. Ocana, Wettability modification of laser-fabricated hierarchical surface structures in Ti-6Al-4V titanium alloy. Appl. Surf. Sci. 463, 838-846 (2019)

38. J. Bonse, R. Koter, M. Hartelt, D. Spaltmann, S. Pentzien, S. Hohm, A. Rosenfeld, J. Kruger, Femtosecond laser-induced periodic surface structures on steel and titanium alloy for tribological applications. Appl. Phys. A 418, 572-579 (2014)

39. J. Byskov-Nielsen, J.M. Savolainen, M.S. Christensen, P. Balling, Ultra-short pulse laser ablation of metals: threshold fluence, incubation coefficient and ablation rates. Appl. Phys. A 101, 97-101 (2010) 